\title{
A New Access Route to Functionalized Cispentacins from Norbornene $\beta$-Amino Acids
}

\author{
Loránd Kiss, ${ }^{[a]}$ Maria Cherepanova, ${ }^{[a]}$ Enikő Forróo, ${ }^{[a]}$ and Ferenc Fülöp*[a, b]
}

\begin{abstract}
An efficient and simple new stereocontrolled access route to novel disubstituted cispentacin derivatives with multiple stereogenic centers from norbornene $\beta$-lactam has been developed. The synthesis involves olefinic bond functionalization by dihydroxylation followed by oxidative ring cleavage and transformation of the dialdehyde intermediate through a Wittig reaction.
\end{abstract}

Keywords: cyclopentanes • enzymatic resolution $\cdot$ oxidation stereochemistry $\cdot$ Wittig reactions

\section{Introduction}

Cyclic $\beta$-amino acids with valuable pharmacological potential have generated increasing interest during the past two decades. These compounds are precursors of bioactive $\beta$-lactams and are important elements of many natural products. A number of derivatives of this class of compounds, such as cispentacin [(1R,2S)-2-aminocyclopentanecarboxylic acid], icofungipen $[(1 R, 2 S)$-2-amino-4-methylenecyclopentanecarboxylic acid], and oryzoxymycin, are strong antifungal agents or antibiotics. ${ }^{[1]}$ The conformationally rigid alicyclic and heterocyclic $\beta$-amino acids are highly important building blocks for the synthesis of peptides to be applied in drug research. ${ }^{[2]}$

Highly functionalized cyclic amino acids have become of great interest in organic and medicinal chemistry research during the past ten years. Cyclohexane amino acids, such as Tamiflu, the O-heterocyclic amino acid Zanamivir and related derivatives, ${ }^{[3,4]}$ and the cyclopentane amino acid Peramivir and its analogues, ${ }^{[5]}$ exert important antiviral activities. Consequently, in view of this enormous pharmacological potential, the synthesis of multisubstituted cycloalkane amino acids has been a focus in synthetic and medicinal chemistry.

The main strategies relating to the synthesis of highly functionalized cyclic amino acids are based on the functionalization of a ring $\mathrm{C}-\mathrm{C}$ double bond or on the ring closure of already substituted carboxylate entities, followed by transformation into amino acids or amino carboxylates. In the framework of the first approach, the most relevant

[a] Dr. L. Kiss, M. Cherepanova, Dr. E. Forró, Prof. F. Fülöp Institute of Pharmaceutical Chemistry, University of Szeged 6720 Szeged, Eötvös u. 6, (Hungary)

E-mail: fulop@pharm.u-szeged.hu

[b] Prof. F. Fülöp

Stereochemistry Research Group of the Hungarian Academy of Sciences, University of Szeged 6720 Szeged, Eötvös u. 6, (Hungary)

Supporting information for this article is available on the WWW under http://dx.doi.org/10.1002/chem.201203183. methods are regio- and stereoselective iodooxazination and iodooxazoline formation, iodolactonization, ${ }^{[6]}$ iodolactamization, ${ }^{[7]}$ stereoselective epoxidation and regioselective oxirane opening ${ }^{[8]}$ stereoselective dihydroxylation ${ }^{[9]}$ and functionalization through 1,3-dipolar cycloaddition. ${ }^{[10]}$ Other strategies include [2+2] cycloaddition, ${ }^{[11]}$ syntheses from substituted cyclic $\beta$-keto esters, ${ }^{[12]}$ ring-opening metathesis, ${ }^{[13]}$ crossmetathesis of the imino esters resulting from fluorinated imidoyl chlorides and ethyl acrylate, ${ }^{[14]}$ and lithium amide promoted conjugate addition to $\alpha, \beta$-unsaturated carboxylates. ${ }^{[15]}$ Very few examples of the synthesis of alkyl-substituted cispentacin derivatives are to be found in the literature. A general method for the preparation of alkylated cyclic $\beta$ amino acid derivatives is the lithium amide mediated conjugate addition to $\alpha, \beta$-unsaturated carboxylates. ${ }^{[15]} \mathrm{A}$ less general approach is the transformation of alkylated bicyclic anhydrides by ammonolysis and Hoffman degradation. ${ }^{[1 \mathrm{~g}]}$

\section{Results and Discussion}

The aim of this work is to describe a novel approach to the preparation of difunctionalized cispentacins from a $\beta$-lactam derived exo-norbornadiene.

The concept of the synthetic route, represented in the retrosynthetic scheme (Scheme 1), was the functionalization of the $\mathrm{C}-\mathrm{C}$ ring double bond of the di-exo-norbornene $\beta$ lactam by stereoselective dihydroxylation, conversion of the vicinal dihydroxylated amino ester by oxidative $\mathrm{C}-\mathrm{C}$ bond

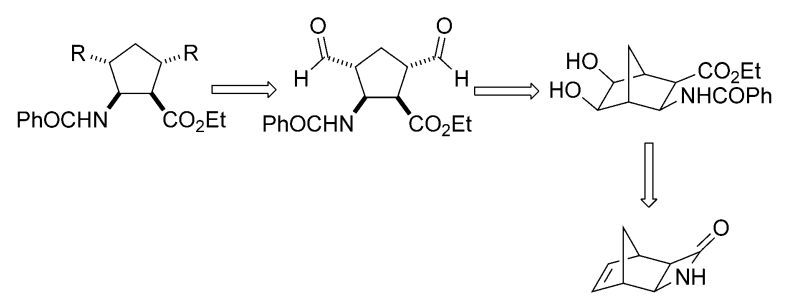

Scheme 1. Retrosynthetic scheme for the preparation of difunctionalized cispentacins from a $\beta$-lactam derived exo-norbornadiene. 
cleavage with ring opening to generate the corresponding dialdehyde derivative, followed by Wittig transformation with different phosphoranylides and reduction of the olefinic bond (Scheme 1).

The initial $\beta$-lactam, 1, was transformed by known procedures ${ }^{[9 c-e]}$ through lactam ring opening with ethanolic $\mathrm{HCl}$ solution, N-benzoylation, and dihydroxylation of amino ester 2 with $N$-methylmorpholine $N$-oxide (NMO) and a catalytic amount of $\mathrm{OsO}_{4}$ into the corresponding vicinal cisdiol 3 (Scheme 2). Bicyclic dihydroxylated amino ester 3

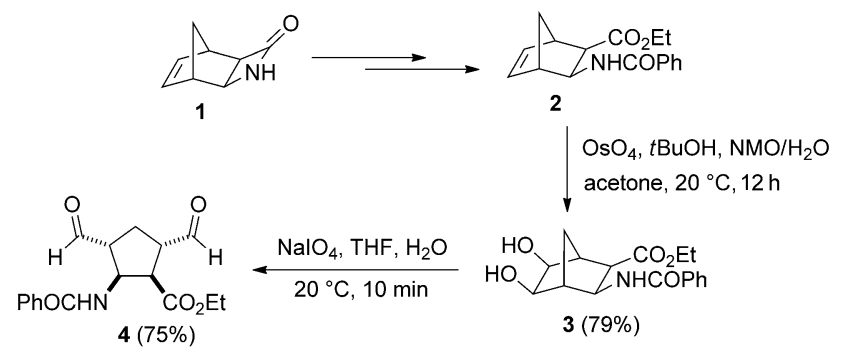

Scheme 2. Synthesis of dialdehyde 4 from $\beta$-lactam 1. THF: tetrahydrofuran.

was next subjected to oxidative $\mathrm{C}-\mathrm{C}$ cleavage with sodium periodate. In contrast with similar transformations of monocyclic dihydroxylated amino esters, in which the corresponding dialdehydes could not be isolated, ${ }^{[9 c-e]}$ the dialdehyde formed in the present case, in which the two formyl moieties are in a trans relationship relative to the carboxylate and amide groups, proved to be a stable, isolable compound (Scheme 2).

Amino ester 4, bearing two formyl substituents, was an excellent precursor for further functionalizations ${ }^{[16]}$ for example, the preparation of alkyl-substituted cispentacins. In order to create a $\mathrm{C}-\mathrm{C}$ double bond, compound 4 was subjected to an in situ Wittig reaction (Scheme 3). Methyltriphenylphosphonium bromide was treated with potassium tert-butoxide in dry THF for $15 \mathrm{~min}$ to furnish the corresponding phosphorane, which was treated immediately with dialdehyde 4 . The reaction resulted in the corresponding dialkenylated $\beta$-aminocyclopentanecarboxylate $\mathbf{5}$ in moderate yield $(51 \%)$. Similarly, compound 4 treated with the phosphorane derived from benzyltriphenylphosphonium bromide gave the corresponding dialkenylated $\beta$-amino ester $\mathbf{6}$ in $34 \%$ yield (Scheme 3 ). Next, amino esters 5 and $\mathbf{6}$ were hydrogenated under catalytic conditions into the corresponding dialkyl-substituted cispentacin derivatives $\mathbf{7}$ and $\mathbf{8}$ in

Abstract in Hungarian: Norbornénvázas $\beta$-laktámból kiindulva sztereokontrollált átalakításokkal négy sztereogén centrumot tartalmazó diszubsztituált ciszpentacin származékokat állítottunk elö. A szintézisút kulcslépései a gyürü szén-szén kettôs kötésének dihidroxilálása, majd oxidatív gyürünyitást követô Wittig reakcióval történö funkcionalizálásai voltak.

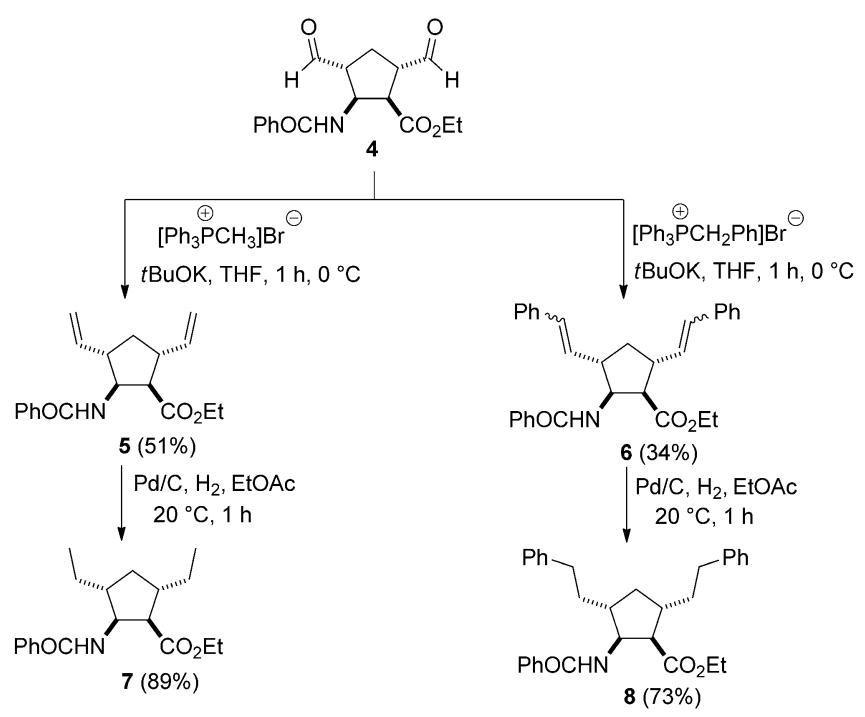

Scheme 3. Synthesis of difunctionalized cispentacin derivatives $\mathbf{7}$ and $\mathbf{8}$ from dialdehyde 4 .

good yields (Scheme 3). It is important to note that the structure of the initial norbornene $\beta$-aminocarboxylate determined the configuration of the newly formed stereogenic centers at positions $\mathrm{C} 3$ and $\mathrm{C} 5$ in these products.

By analogous synthetic procedures, novel substituted cispentacin derivatives were prepared by using various commercially available phosphoranes. When dialdehyde 4 was treated with methyl (triphenylphosphoranylidene)acetate in THF (Scheme 4), the corresponding Wittig product 9, with two $\mathrm{C}-\mathrm{C}$ double bonds, was formed in relatively good yield $(74 \%)$. In a similar transformation, amino ester 4 was treated with (triphenylphosphoranylidene)-2-propanone in THF to give the olefinic product $\mathbf{1 0}$. Compounds 9 and $\mathbf{1 0}$ were

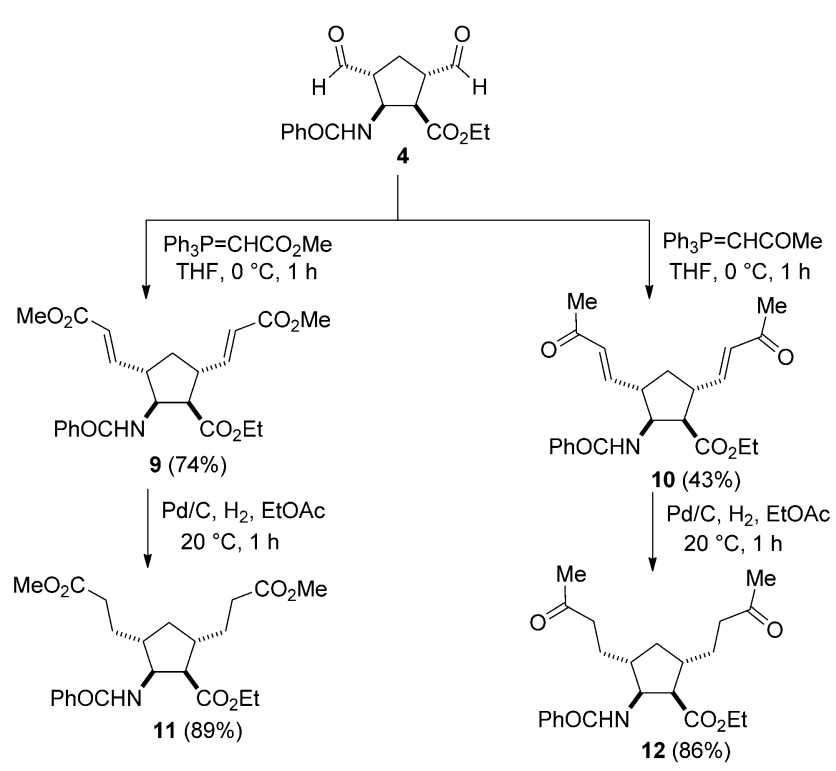

Scheme 4. Synthesis of difunctionalized cispentacin derivatives $\mathbf{1 1}$ and $\mathbf{1 2}$ from dialdehyde 4 . 
then subjected to hydrogenolysis in the presence of a catalytic amount of $\mathrm{Pd} / \mathrm{C}$ to furnish the corresponding difunctionalized cispentacin derivatives $\mathbf{1 1}$ and $\mathbf{1 2}$ (Scheme 4).

The newly developed protocol described above for the synthesis of functionalized cispentacins allowed the preparation of these compounds in enantiomerically pure form. Hence, racemic $\beta$-lactam 1 was subjected to enantioselective lactam ring opening by a facile, solvent-free enzymatic method, ${ }^{[17]}$ which was scaled up successfully (Scheme 5).

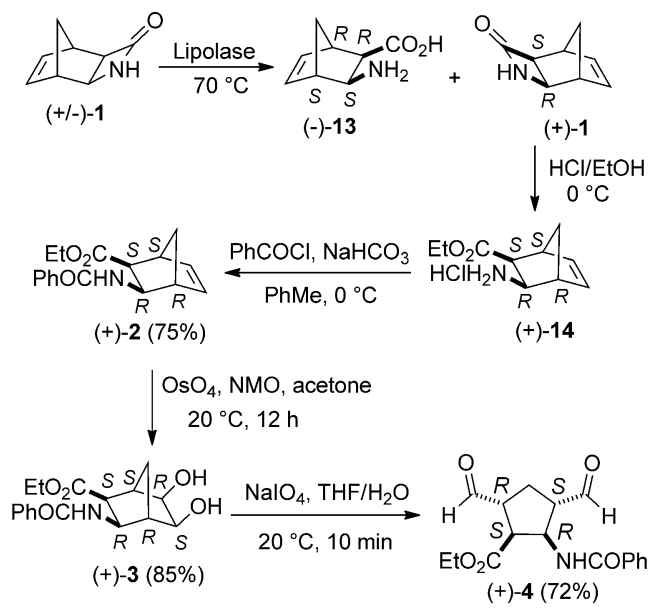

Scheme 5. Enzymatic resolution of racemic $\beta$-lactam $\mathbf{1}$ and synthesis of enantiomerically pure dialdehyde $\mathbf{4}$.

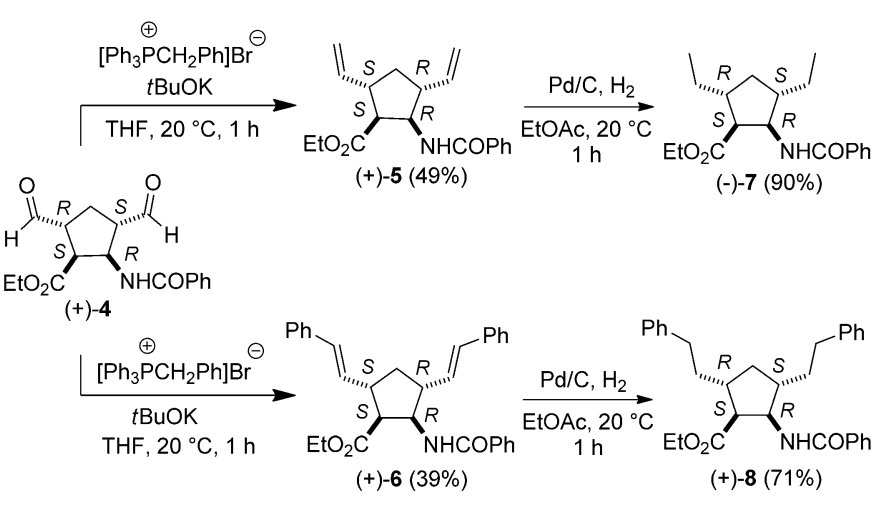

Scheme 6. Synthesis of optically active difunctionalized cispentacin derivatives (-)-7 and (+)-8 from dialdehyde (+)-4.

In conclusion, we have developed a novel synthetic access route to difunctionalized cispentacin derivatives from a diexo-norbornene $\beta$-amino acid through dihydroxylation, periodate oxidation involving ring cleavage, and conversion of the dialdehyde derivative formed into the functionalized derivatives through a Wittig reaction. The structure of the initial compound determines the stereochemistry of the newly formed stereogenic centers on the cyclopentane framework of the products. The developed method based on oxidative ring cleavage and dialdehyde-intermediate transformations under different conditions might be generalized and applied toward the synthesis of a variety of substituted cispentacin
Lactam 1 was mixed well with Lipolase, water was added, and the mixture was shaken in an incubator shaker at $70^{\circ} \mathrm{C}$. The resulting enantiomerically pure amino acid (-)-13 (ee>98\%) and unreacted $\beta$-lactam $(+)-\mathbf{1}$ $(e e=99 \%)$ were easily separated (see the Experimental Section). Next, treatment of enantiomer (+)-1 with $\mathrm{HCl} / \mathrm{EtOH}$, followed by benzoylation, afforded optically pure (+)-2, which, after dihydroxylation and $\mathrm{C}-\mathrm{C}$ bond cleavage, led to dialdehyde (+)-4 (Scheme 5).

The in situ Wittig reaction of $(+)-4$ furnished olefinic derivatives (+)-5 and (+)-6, after which $\mathrm{C}-\mathrm{C}$ double-bond saturation by catalytic hydrogenation led to substituted cispentacin derivatives (-)-7 and (+)-8 in optically active forms (Scheme 6).

The reaction of diformyl amino ester (+)-4 with methyl (triphenylphosphoranylidene)acetate or (triphenylphosphoranylidene)-2-propanone afforded enantiomers (+)-9 and $(+)-\mathbf{1 0}$, respectively, the hydrogenation of which furnished $\beta$-amino acid derivatives $(+)-\mathbf{1 1}$ and $(+)-\mathbf{1 2}$ in enantiomerically pure form (Scheme 7). derivatives. The synthetic procedure was extended to the preparation of these substances in enantiomerically pure form.

\section{Experimental Section}

General information: The chemicals were purchased from Sigma-Aldrich. The NMR spectra were recorded at $400 \mathrm{MHz}$ with $\mathrm{CDCl}_{3}$ or $\left[\mathrm{D}_{6}\right]$ dimethylsulfoxide $\left(\left[\mathrm{D}_{6}\right] \mathrm{DMSO}\right)$ as the solvent and tetramethylsilane as an internal standard. The solvents were used as received from the suppli- 
ers. Melting points were determined with a Kofler apparatus. Elemental analyses were recorded on a Perkin-Elmer CHNS-2400 Ser II elemental analyzer. Silica gel 60 F254 was purchased from Merck. Mass spectra were recorded on a Finnigan MAT 95S spectrometer.

General procedure for dihydroxylation of $\mathbf{N}$-protected amino esters: ${ }^{[9]}$ $\mathrm{OsO}_{4}(0.5 \mathrm{~mL}, 0.03 \mathrm{mmol})$ in $t \mathrm{BuOH}(0.06 \mathrm{M})$ was added to a solution of N-protected $\beta$-amino ester $( \pm)-2(2 \mathrm{~g}, 7.72 \mathrm{mmol})$ and NMO $(3 \mathrm{~mL}$, $29.1 \mathrm{mmol})$ in acetone $(40 \mathrm{~mL})$, and the resulting mixture was stirred for $12 \mathrm{~h}$ at room temperature. After completion of the reaction, as monitored by TLC, saturated aqueous $\mathrm{Na}_{2} \mathrm{SO}_{3}$ solution $(120 \mathrm{~mL})$ was added, and the reaction mixture was extracted with $\mathrm{CH}_{2} \mathrm{Cl}_{2}(3 \times 50 \mathrm{~mL})$. The combined organic phases were dried over $\mathrm{Na}_{2} \mathrm{SO}_{4}$, filtered, and evaporated in vacuo. The crude product was purified by means of column chromatography on silica gel ( $n$-hexane/EtOAc 1:4).

General procedure for oxidative $\mathrm{C}$-C cleavage (dialdehyde formation): $\mathrm{NaIO}_{4}(269 \mathrm{mg}, 1.26 \mathrm{mmol})$ was added to a solution of dihydroxylated amino ester $( \pm)-3(200 \mathrm{mg}, 0.63 \mathrm{mmol})$ in $\mathrm{THF} / \mathrm{H}_{2} \mathrm{O}(11 \mathrm{~mL}, 10: 1)$, and reaction mixture was stirred for $10 \mathrm{~min}$ at room temperature under an $\mathrm{Ar}$ atmosphere. The mixture was then quenched by addition of water $(20 \mathrm{~mL})$ and extracted with $\mathrm{CH}_{2} \mathrm{Cl}_{2}(2 \times 15 \mathrm{~mL})$. The combined organic phases were dried over $\mathrm{Na}_{2} \mathrm{SO}_{4}$, filtered, and evaporated in vacuo. The crude material was purified by column chromatography on silica gel $(n-$ hexane/EtOAc 1:4).

Ethyl $\left(1 R^{*}, 2 S^{*}, 3 R^{*}, 5 S^{*}\right)$-2-benzamido-3,5-diformylcyclopentanecarboxylate (4): White solid; yield: $75 \%$; m.p. $114-116^{\circ} \mathrm{C} ; R_{\mathrm{f}}=0.4$ ( $n$-hexane/ EtOAc 1:4); ${ }^{1} \mathrm{H}$ NMR $\left(\mathrm{CDCl}_{3}, 400 \mathrm{MHz}\right): \delta=1.26(\mathrm{t}, J=7.11 \mathrm{~Hz}, 3 \mathrm{H}$; $\left.\mathrm{CH}_{3}\right), 2.22-2.40\left(\mathrm{~m}, 2 \mathrm{H} ; \mathrm{CH}_{2}\right), 3.13-3.21(\mathrm{~m}, 1 \mathrm{H} ; \mathrm{H} 5), 3.33-3.40(\mathrm{~m}, 1 \mathrm{H}$; $\mathrm{H} 3), 3.47-3.51(\mathrm{~m}, 1 \mathrm{H} ; \mathrm{H} 1), 4.17-4.25\left(\mathrm{~m}, 2 \mathrm{H} ; \mathrm{OCH}_{2}\right), 4.78-4.83(\mathrm{~m}$, $1 \mathrm{H} ; \mathrm{H} 2), 7.39-7.78(\mathrm{~m}, 5 \mathrm{H} ; \mathrm{ArH}), 9.72-9.78 \mathrm{ppm}(\mathrm{m}, 2 \mathrm{H} ; \mathrm{COH})$; ${ }^{13} \mathrm{C}$ NMR ([D 6 DMSO, $\left.400 \mathrm{MHz}\right): \delta=14.7,24.6,48.4,52.2,52.5,55.7$, 61.2, 128.2, 129.0, 132.1, 135.2, 167.1, 171.6, 202.5, 202.8 ppm; MS (ESI): $\mathrm{m} / \mathrm{z} 318.67[M+1]$; elemental analysis: calcd (\%) for $\mathrm{C}_{17} \mathrm{H}_{19} \mathrm{NO}_{5}$ : C 64.34, H 6.03, N 4.41; found: C 64.21, H 6.40, N 4.64.

\section{General procedures for Wittig reaction :}

Procedure A: The dialdehyde derivative $( \pm)-4(200 \mathrm{mg}, 0.63 \mathrm{mmol})$ was dissolved in dry THF $(5 \mathrm{~mL})$, and the corresponding Wittig reagent $(1.26 \mathrm{mmol})$ was added to the solution. After being stirred for $1 \mathrm{~h}$ at room temperature, the reaction mixture was concentrated in vacuo and purified by column chromatography on silica gel ( $n$-hexane/EtOAc).

Procedure B: The Wittig reagent was prepared first by adding $t \mathrm{BuOK}$ $(1.26 \mathrm{mmol})$ to a solution of the corresponding phosphonium salt $(1.26 \mathrm{mmol})$ in dry THF $(5 \mathrm{~mL})$, and the mixture was stirred at $0^{\circ} \mathrm{C}$ for $10 \mathrm{~min}$. The dialdehyde derivative $( \pm)-4(200 \mathrm{mg}, 0.63 \mathrm{mmol})$ was dissolved in dry THF ( $5 \mathrm{~mL}$ ) and added dropwise to the solution of the in situ generated Wittig reagent mixture. After being stirred for $1 \mathrm{~h}$ at room temperature, the reaction mixture was concentrated in vacuo and purified by column chromatography on silica gel ( $n$-hexane/EtOAc).

Ethyl $\left(1 R^{*}, 2 S^{*}, 3 S^{*}, 5 R^{*}\right)$-2-benzamido-3,5-divinylcyclopentanecarboxylate (5): White solid; yield: $51 \%$; m.p. $92-94^{\circ} \mathrm{C} ; R_{\mathrm{f}}=0.6(n$-hexane/ EtOAc 3:1); ${ }^{1} \mathrm{H}$ NMR $\left(\mathrm{CDCl}_{3}, 400 \mathrm{MHz}\right): \delta=1.16(\mathrm{t}, J=7.08 \mathrm{~Hz}, 3 \mathrm{H}$; $\left.\mathrm{CH}_{3}\right), 1.41-1.59\left(\mathrm{~m}, 1 \mathrm{H} ; \mathrm{CH}_{2}\right), 2.09-2.17\left(\mathrm{~m}, 1 \mathrm{H} ; \mathrm{CH}_{2}\right), 2.68-2.78(\mathrm{~m}$, $1 \mathrm{H}$; H1), 2.95-3.08 (m, 2H; H3, H5), 4.02-4.18 (m, 2H; $\left.\mathrm{OCH}_{2}\right), 4.57-$ $4.60(\mathrm{~m}, 1 \mathrm{H} ; \mathrm{H} 2), 4.98-5.16(\mathrm{~m}, 4 \mathrm{H} ; \mathrm{C}=\mathrm{C}-\mathrm{H}), 5.76-5.88(\mathrm{~m}, 2 \mathrm{H} ; \mathrm{C}=\mathrm{C}-$ $\mathrm{H}), 6.71-6.88(\mathrm{~m}, 1 \mathrm{H} ; \mathrm{NH}), 7.35-7.80 \mathrm{ppm}(\mathrm{m}, 5 \mathrm{H} ; \mathrm{ArH}) ;{ }^{13} \mathrm{C} \mathrm{NMR}$ ([D $\left.\left.\mathrm{D}_{6}\right] \mathrm{DMSO}, 400 \mathrm{MHz}\right): \delta=14.7,37.1,42.7,45.7,53.7,56.7,60.6,115.4$, $115.9,128.3,128.8,135.3,138.5,140.4,141.2,167.0,172.7 \mathrm{ppm}$; MS (ESI): $\mathrm{m} / \mathrm{z} 314.42[M+1]$; elemental analysis: calcd $(\%)$ for $\mathrm{C}_{19} \mathrm{H}_{23} \mathrm{NO}_{3}: \mathrm{C} 72.82$, H 7.40, N 4.47; found: C 72.55, H 7.21, N 4.64.

Ethyl $\left(1 R^{*}, 2 S^{*}, 3 S^{*}, 5 R^{*}\right)$-2-benzamido-3,5-distyrylcyclopentanecarboxylate (6): White solid; yield: $34 \%$; m.p. $120-123^{\circ} \mathrm{C} ; R_{\mathrm{f}}=0.2(n$-hexane/ EtOAc 3:1); ${ }^{1} \mathrm{H}$ NMR ([D 6$]$ DMSO, $\left.400 \mathrm{MHz}\right): \delta=0.96(\mathrm{t}, J=7.06 \mathrm{~Hz}$, $\left.3 \mathrm{H} ; \mathrm{CH}_{3}\right), 1.58-1.62\left(\mathrm{~m}, 1 \mathrm{H} ; \mathrm{CH}_{2}\right), 2.13-2.21\left(\mathrm{~m}, 1 \mathrm{H} ; \mathrm{CH}_{2}\right), 3.02-3.16$ (m, 2H; H3, H5), 3.25-3.29 (m, 1H; H1), 3.89-3.93 (m, 2H; $\left.\mathrm{OCH}_{2}\right)$, 4.63-4.66 (m, 1H; H2), 6.28-6.53 (m, 4H; $\mathrm{C}=\mathrm{C}-\mathrm{H}), 7.16-7.81(\mathrm{~m}, 15 \mathrm{H}$; $\mathrm{ArH}), 8.44 \mathrm{ppm}$ (brs, $1 \mathrm{H} ; \mathrm{NH}) ;{ }^{13} \mathrm{C}$ NMR ([D $]$ DMSO, $\left.400 \mathrm{MHz}\right): \delta=$ $14.8,38.3,45.4,48.2,54.3,57.4,60.8,126.8,128.2,129.0,129.4,130.3$, 130.7, 132.0, 132.4, 133.1, 135.3, 137.8, 167.1, $172.8 \mathrm{ppm}$; MS (ESI): $\mathrm{m} / \mathrm{z}$
$466.85[M+1]$; elemental analysis: calcd (\%) for $\mathrm{C}_{31} \mathrm{H}_{31} \mathrm{NO}_{3}: \mathrm{C} 79.97, \mathrm{H}$ 6.71, N 3.01; found: C 79.64, H 6.41, N 3.32 .

Dimethyl $3,3^{\prime}-\left(\left(1 R^{*}, 3 S^{*}, 4 S^{*}, 5 R^{*}\right)\right.$-4-benzamido-5-(ethoxycarbonyl)cyclopentane-1,3-diyl)diacrylate (9): White solid; yield: $74 \%$; m.p. $152-154^{\circ} \mathrm{C}$; $R_{\mathrm{f}}=0.45(n$-hexane/EtOAc $1: 1) ;{ }^{1} \mathrm{H} \mathrm{NMR}\left(\mathrm{CDCl}_{3}, 400 \mathrm{MHz}\right): \delta=1.16(\mathrm{t}$, $\left.J=7.16 \mathrm{~Hz}, 3 \mathrm{H} ; \mathrm{CH}_{3}\right), 1.53-1.63\left(\mathrm{~m}, 2 \mathrm{H} ; \mathrm{CH}_{2}\right), 2.89-3.00(\mathrm{~m}, 1 \mathrm{H} ; \mathrm{H} 5)$, $3.10-3.25(\mathrm{~m}, 2 \mathrm{H} ; \mathrm{H} 1, \mathrm{H} 3), 3.70\left(\mathrm{~s}, 3 \mathrm{H} ; \mathrm{OCH}_{3}\right), 3.75\left(\mathrm{~s}, 3 \mathrm{H} ; \mathrm{OCH}_{3}\right)$, $4.02-4.18\left(\mathrm{~m}, 2 \mathrm{H} ; \mathrm{OCH}_{2}\right), 4.72(\mathrm{q}, J=8.99 \mathrm{~Hz}, 1 \mathrm{H} ; \mathrm{H} 4), 5.88-5.92(\mathrm{~m}$, $2 \mathrm{H} ; \mathrm{C}=\mathrm{C}-\mathrm{H}), 6.75(\mathrm{brs}, 1 \mathrm{H} ; \mathrm{NH}), 6.87-6.97(\mathrm{~m}, 2 \mathrm{H} ; \mathrm{C}=\mathrm{C}-\mathrm{H}), 7.37-$ $7.79 \mathrm{ppm}(\mathrm{m}, 5 \mathrm{H} ; \mathrm{ArH}) ;{ }^{13} \mathrm{C}$ NMR ([D $]$ DMSO, $\left.400 \mathrm{MHz}\right): \delta=14.8,36.1$, 44.2, 47.2, 52.2, 53.1, 56.2, 60.9, 121.6, 121.9, 128.1, 129.0, 132.1, 135.0, 150.1, 150.8, 166.8, 167.0, $172.2 \mathrm{ppm}$; MS (ESI): $\mathrm{m} / \mathrm{z} 430.42[M+1]$; elemental analysis: calcd (\%) for $\mathrm{C}_{23} \mathrm{H}_{27} \mathrm{NO}_{7}$ : C 64.32, H 6.34, N 3.26; found: C 64.68, H 6.01, N 3.01.

Ethyl $\left(1 R^{*}, 2 S^{*}, 3 S^{*}, 5 R^{*}\right)$-2-benzamido-3,5-di(3'-oxobut-1-enyl)cyclopentanecarboxylate (10): Yellow solid; yield: $43 \%$; m.p. $110-113^{\circ} \mathrm{C} ; R_{\mathrm{f}}=0.3$ $\left(n\right.$-hexane/EtOAc 1:1); ${ }^{1} \mathrm{H}$ NMR $\left(\mathrm{CDCl}_{3}, 400 \mathrm{MHz}\right): \delta=1.18(\mathrm{t}, J=$ $\left.7.22 \mathrm{~Hz}, 3 \mathrm{H} ; \mathrm{CH}_{3}\right), 1.55-1.70\left(\mathrm{~m}, 2 \mathrm{H} ; \mathrm{CH}_{2}\right), 2.20-2.31\left(\mathrm{~m}, 6 \mathrm{H} ; 2 \mathrm{CH}_{3}\right.$, 2.91-3.03 (m, 1H; H1), 3.11-3.27 (m, 2H; H3, H5), 4.05-4.20 (m, 2H; $\left.\mathrm{OCH}_{2}\right), 4.73-4.76(\mathrm{~m}, 1 \mathrm{H} ; \mathrm{H} 2), 6.11-6.15(\mathrm{~m}, 2 \mathrm{H} ; \mathrm{C}=\mathrm{C}-\mathrm{H}), 6.69-6.80$ $(\mathrm{m}, 2 \mathrm{H} ; \mathrm{C}=\mathrm{C}-\mathrm{H}), 6.90($ brs, $1 \mathrm{H} ; \mathrm{NH}), 7.40-7.75 \mathrm{ppm}(\mathrm{m}, 5 \mathrm{H} ; \mathrm{ArH})$; ${ }^{13} \mathrm{C}$ NMR ([D 6 DMSO, $\left.400 \mathrm{MHz}\right): \delta=14.7,27.6,36.5,44.3,47.5,53.4$, 56.7, 61.0, 128.1, 129.0, 131.4, 131.8, 132.1, 135.0, 148.9, 149.5, 167.0, 172.1, $198.8 \mathrm{ppm}$; MS (ESI): $\mathrm{m} / \mathrm{z} 398.67$ [M+1]; elemental analysis: calcd (\%) for $\mathrm{C}_{23} \mathrm{H}_{27} \mathrm{NO}_{5}$ : C 69.50, H 6.85, N 3.52; found: C 69.28, H 6.53, N 3.16 .

General procedure for saturation of the olefinic bond: A solution of olefinic compound $( \pm)-\mathbf{5},( \pm)-\mathbf{6},( \pm)-\mathbf{9}$, or $( \pm)-\mathbf{1 0}(100 \mathrm{mg}, 0.32 \mathrm{mmol})$ and $\mathrm{Pd} / \mathrm{C}(20 \mathrm{mg}, 10 \mathrm{~mol} \%)$ in EtOAc $(20 \mathrm{~mL})$ was stirred under a $\mathrm{H}_{2}$ atmosphere for $1 \mathrm{~h}$. The reaction mixture was then filtered through silica gel and celite. The filtrate was concentrated under reduced pressure and purified by column chromatography on silica gel ( $n$-hexane/EtOAc).

Ethyl $\left(1 R^{*}, 2 S^{*}, 3 R^{*}, 5 S^{*}\right)$-2-benzamido-3,5-diethylcyclopentanecarboxylate (7): White solid; yield: $89 \%$; m.p. $74-77^{\circ} \mathrm{C} ; R_{\mathrm{f}}=0.7$ ( $n$-hexane/ EtOAc 3:1); ${ }^{1} \mathrm{H}$ NMR $\left(\mathrm{CDCl}_{3}, 400 \mathrm{MHz}\right): \delta=0.93(\mathrm{t}, J=7.44 \mathrm{~Hz}, 6 \mathrm{H}$; $\mathrm{H} 1, \mathrm{H} 9), 1.18\left(\mathrm{t}, J=7.04 \mathrm{~Hz}, 3 \mathrm{H} ; \mathrm{CH}_{3}\right), 1.24-1.70\left(\mathrm{~m}, 5 \mathrm{H} ; \mathrm{CH}_{2}\right), 1.93$ $2.03\left(\mathrm{~m}, 1 \mathrm{H} ; \mathrm{CH}_{2}\right), 2.12-2.24(\mathrm{~m}, 2 \mathrm{H} ; \mathrm{H} 3$ and $\mathrm{H} 5), 2.81-2.87(\mathrm{~m}, 1 \mathrm{H}$; $\mathrm{H} 1), 4.00-4.18\left(\mathrm{~m}, 2 \mathrm{H} ; \mathrm{OCH}_{2}\right), 4.40-4.43(\mathrm{~m}, 1 \mathrm{H} ; \mathrm{H} 2), 6.77(\mathrm{brs}, 1 \mathrm{H}$; $\mathrm{NH}), 7.38-7.81 \mathrm{ppm}(\mathrm{m}, 5 \mathrm{H} ; \mathrm{ArH}) ;{ }^{13} \mathrm{C}$ NMR ([D $]$ DMSO, $\left.400 \mathrm{MHz}\right)$ : $\delta=13.1,14.7,26.9,28.5,36.9,43.3,45.9,54.3,57.4,60.4,128.2,128.9$, 131.9, 135.5, 167.0, $173.8 \mathrm{ppm}$; MS (ESI): $\mathrm{m} / z 318.33$ [M+1]; elemental analysis: calcd (\%) for $\mathrm{C}_{19} \mathrm{H}_{27} \mathrm{NO}_{3}$ : C 71.89, $\mathrm{H}$ 8.57, N 4.41; found: $\mathrm{C}$ 71.58, H 8.30, N 4.16.

Ethyl $\left(1 R^{*}, 2 S^{*}, 3 R^{*}, 5 S^{*}\right)$-2-benzamido-3,5-diphenethylcyclopentanecarboxylate (8): White solid; yield: $73 \%$; m.p. $63-66^{\circ} \mathrm{C} ; R_{\mathrm{f}}=0.3$ ( $n$-hexane/ EtOAc 3:1); ${ }^{1} \mathrm{H}$ NMR ([D 6 DMSO, $\left.400 \mathrm{MHz}\right): \delta=1.19$ (t, $J=7.17 \mathrm{~Hz}$, $\left.3 \mathrm{H} ; \mathrm{CH}_{3}\right), 1.55-2.15\left(\mathrm{~m}, 8 \mathrm{H} ; \mathrm{CH}_{2}\right), 2.61-2.81\left(\mathrm{~m}, 4 \mathrm{H} ; \mathrm{CH}_{2}, \mathrm{H} 3, \mathrm{H} 5\right)$, 2.92-2.97 (m, 1H; H1), 4.03-4.19 (m, 2H; $\left.\mathrm{OCH}_{2}\right), 4.53-4.56(\mathrm{~m}, 1 \mathrm{H}$; $\mathrm{H} 2), 6.77$ (brs, $1 \mathrm{H} ; \mathrm{NH}), 7.14-7.80 \mathrm{ppm}(\mathrm{m}, 15 \mathrm{H} ; \mathrm{ArH}) ;{ }^{13} \mathrm{C} \mathrm{NMR}$ ([D $]$ DMSO, $400 \mathrm{MHz}): \delta=14.8,34.5,34.6,36.3,37.4,37.5,41.1,44.3$ 54.5, 57.6, 60.6, 126.4, 126.5, 128.1, 128.9, 129.0, 129.1, 131.9, 135.5, 142.8, 143.1, 167.1, $173.8 \mathrm{ppm}$; MS (ESI): $\mathrm{m} / \mathrm{z} 470.50$ [M+1]; elemental analysis: calcd (\%) for $\mathrm{C}_{31} \mathrm{H}_{35} \mathrm{NO}_{3}$ : C 79.28, $\mathrm{H} 7.51, \mathrm{~N} 2.98$; found: $\mathrm{C} 78.92, \mathrm{H}$ $7.21, \mathrm{~N} 2.61$.

Dimethyl $3,3^{\prime}-\left(\left(1 S^{*}, 3 R^{*}, 4 S^{*}, 5 R^{*}\right)\right.$-4-benzamido-5-(ethoxycarbonyl)cyclopentane-1,3-diyl)dipropanoate (11): Yellowish-white solid; yield: $89 \%$; m.p. $\quad 91-92^{\circ} \mathrm{C} ; \quad R_{\mathrm{f}}=0.3 \quad(n$-hexane/EtOAc $1: 1) ;{ }^{1} \mathrm{H} \mathrm{NMR} \quad\left(\mathrm{CDCl}_{3}\right.$, $400 \mathrm{MHz}): \delta=1.16\left(\mathrm{t}, J=7.05 \mathrm{~Hz}, 3 \mathrm{H} ; \mathrm{CH}_{3}\right), 1.55-2.46\left(\mathrm{~m}, 12 \mathrm{H} ; \mathrm{CH}_{2}\right.$ $\mathrm{H} 3, \mathrm{H} 5), 2.84-2.90(\mathrm{~m}, 1 \mathrm{H} ; \mathrm{H} 1), 3.63\left(\mathrm{~s}, 3 \mathrm{H} ; \mathrm{OCH}_{3}\right), 3.67(\mathrm{~s}, 3 \mathrm{H}$; $\left.\mathrm{OCH}_{3}\right), 4.00-4.14\left(\mathrm{~m}, 2 \mathrm{H} ; \mathrm{OCH}_{2}\right), 4.41-4.44(\mathrm{~m}, 1 \mathrm{H} ; \mathrm{H} 2), 6.85(\mathrm{brs}, 1 \mathrm{H}$ $\mathrm{NH}), 7.39-7.52 \mathrm{ppm}(\mathrm{m}, 5 \mathrm{H} ; \mathrm{ArH}) ;{ }^{13} \mathrm{C}$ NMR $\left(\left[\mathrm{D}_{6}\right] \mathrm{DMSO}, 400 \mathrm{MHz}\right)$ : $\delta=14.7,29.3,30.5,32.7,32.8,37.0,41.0,44.1,52.0,54.1,57.2,60.5,128.1$, 128.9, 131.9, 135.4, 166.9, 173.4, 173.9, $174.0 \mathrm{ppm}$; MS (ESI): $\mathrm{m} / \mathrm{z} 434.42$ $[M+1]$; elemental analysis: calcd (\%) for $\mathrm{C}_{23} \mathrm{H}_{31} \mathrm{NO}_{7}: \mathrm{C} 63.73, \mathrm{H} 7.21, \mathrm{~N}$ 3.23; found: C 63.41, H 7.50, N 2.98

Ethyl $\left(1 R^{*}, 2 S^{*}, 3 R^{*}, 5 S^{*}\right)$-2-benzamido-3,5-di(3'-oxobutyl)cyclopentanecarboxylate (12): Yellow oil; yield: $86 \% ; R_{\mathrm{f}}=0.4(n$-hexane/EtOAc $1: 4)$ ${ }^{1} \mathrm{H} \mathrm{NMR}\left(\mathrm{CDCl}_{3}, 400 \mathrm{MHz}\right): \delta=1.20\left(\mathrm{t}, J=7.05 \mathrm{~Hz}, 3 \mathrm{H} ; \mathrm{CH}_{3}\right), 1.52-1.96$ 
(m, 6H; $\left.\mathrm{CH}_{2}\right), 2.12\left(\mathrm{~s}, 3 \mathrm{H} ; \mathrm{CH}_{3}\right), 2.20\left(\mathrm{~s}, 3 \mathrm{H} ; \mathrm{CH}_{3}\right), 2.45-2.62(\mathrm{~m}, 4 \mathrm{H}$; $\left.\mathrm{CH}_{2}, \mathrm{H} 3, \mathrm{H} 5\right), 2.87-2.90$ (m, $\left.1 \mathrm{H} ; \mathrm{H} 1\right), 4.05-4.20$ (m, 2H; $\left.\mathrm{OCH}_{2}\right), 4.40-$ 4.44 (m, 1H; H2), 6.90 (brs, $1 \mathrm{H} ; \mathrm{NH}), 7.40-7.85 \mathrm{ppm}(\mathrm{m}, 5 \mathrm{H} ; \mathrm{ArH})$; ${ }^{13} \mathrm{C}$ NMR ([D $]$ DMSO, $400 \mathrm{MHz}$ ): $\delta=14.8,28.0,29.3,30.5,37.3,41.0$, 42.0, 42.1, 44.0, 54.2, 57.3, 60.5, 128.1, 128.9, 131.9, 135.4, 166.9, 173.6, 208.9, $209.1 \mathrm{ppm}$; MS (ESI): $m / z$ 402.42 [M+1]; elemental analysis: calcd (\%) for $\mathrm{C}_{23} \mathrm{H}_{31} \mathrm{NO}_{5}$ : C 68.80, H 7.78, N 3.49; found: C 68.59, H 7.53, N 3.20 .

Preparative-scale resolution of exo-3-azatricyclo[4.2.1.0 ${ }^{2.5}$ non-7-en-4-one $(( \pm)-1)$ : Crystalline racemic $\mathbf{1}(2 \mathrm{~g}, 14.8 \mathrm{mmol})$ was mixed well with Lipolase (lipase B from Candida antarctica, produced by submerged fermentation of a genetically modified Aspergillus oryzae microorganism and immobilized on a macroporous resin, purchased from Sigma-Aldrich; $6 \mathrm{~g}$; the possibility of reusing the enzyme in several cycles with $E>200$ and only a slight decrease in catalytic activity makes the process an economical one). Water ( $136 \mu \mathrm{L}, 7.52 \mathrm{mmol})$ was added as a nucleophile, and the mixture was shaken in an incubator shaker at $70^{\circ} \mathrm{C}$ for 7 days. The unreacted $\beta$-lactam $(+)-\mathbf{1}$ was washed off the surface of the enzyme with EtOAc $(4 \times 25 \mathrm{~mL})$. Then $\beta$-amino acid $(-)-\mathbf{1 3}$ was washed out with distilled water $(4 \times 25 \mathrm{~mL})$. The easy separation of the products is due to the solubility of the lactam in EtOAc and the solubility of the amino acid in water, whereas the enzyme is not soluble in EtOAc or water. $(1 R, 2 R, 5 S, 6 S)-\beta$-lactam $((+)-\mathbf{1})$ : yield: $917 \mathrm{mg}, 46 \% ;[\alpha]_{D}^{25}=+121.1(c=$ $\left.0.5 ; \mathrm{CHCl}_{3}\right)$; m.p. $94-95^{\circ} \mathrm{C} ; e e=99 \%$. $(1 R, 2 R, 3 S, 4 S)-\beta$-amino acid $((-)$ 13): yield: $1.02 \mathrm{~g}, 45 \%$; $[\alpha]_{\mathrm{D}}^{25}=-12.6\left(c=0.5 ; \mathrm{H}_{2} \mathrm{O}\right)$; m.p. $>260^{\circ} \mathrm{C}$; ee $>$ $98 \%$. The ${ }^{1} \mathrm{H}$ NMR spectroscopic data for the products were identical with those given in the literature. ${ }^{[18]}$ The $e e$ value for $(+)-\mathbf{1}$ was determined by using a gas chromatograph equipped with a CP-Chirasil-Dex $\mathrm{CB}$ column $\left(120^{\circ} \mathrm{C}\right.$ for $4 \mathrm{~min} \rightarrow 170^{\circ} \mathrm{C}$ (temperature rise: $10^{\circ} \mathrm{Cmin}^{-1}$; $140 \mathrm{kPa})$ ): retention time for (+)-1: $10.72 \mathrm{~min}$ (antipode: $10.55 \mathrm{~min})$. The $e e$ value for (-)-13 was determined by using same GC column, but after double derivatization ${ }^{[19]}\left(120^{\circ} \mathrm{C}\right.$ for $7 \mathrm{~min} \rightarrow 170^{\circ} \mathrm{C}$ (temperature rise: $\left.10^{\circ} \mathrm{Cmin}^{-1} ; 70 \mathrm{kPa}\right)$ ): retention time for $(-)-13: 14.66 \mathrm{~min}$ (antipode: $14.86 \mathrm{~min})$.

Characterization of the enantiomeric products: All ${ }^{1} \mathrm{H}$ NMR spectra recorded for the enantiomeric substances were the same as for the corresponding racemic counterparts. The ee values were determined by HPLC (Chiralpack IA column, eluent: $n$-hexane/IPA (80:20), flow rate: $0.5 \mathrm{~mL} \mathrm{~min}^{-1}$, detection at $260 \mathrm{~nm}$ ).

Ethyl (1S,2S,3R,4R)-3-benzamidobicyclo[2.2.1]hept-5-ene-2-carboxylate $((+)-2)$ : White solid; yield: $75 \% ;[\alpha]_{\mathrm{D}}^{25}=+35(c=0.23, \mathrm{EtOH})$.

Ethyl (1S,2S,3R,4R,5S,6R)-3-benzamido-5,6-dihydroxybicyclo[2.2.1]heptane-2-carboxylate $((+)-3)$ : White solid; yield: $85 \% ;[\alpha]_{\mathrm{D}}^{25}=+18.5(c=$ $0.325, \mathrm{EtOH}) ; e e=97 \% ; t_{\mathrm{R}}=15.57 \mathrm{~min}$ (antipode: $14.69 \mathrm{~min}$ ).

Ethyl (1S,2R,3S,5R)-2-benzamido-3,5-diformylcyclopentanecarboxylate ((+)-4): White solid; yield: $72 \% ;[\alpha]_{\mathrm{D}}^{25}=+29(c=0.29$, EtOH $)$.

Ethyl (1S,2R,3R,5S)-2-benzamido-3,5-divinylcyclopentanecarboxylate $((+)-5)$ : White solid; yield: $49 \%$; $[\alpha]_{\mathrm{D}}^{25}=+29.5(c=0.25, \mathrm{EtOH}) ; e e=$ $96 \% ; t_{\mathrm{R}}=12.51 \mathrm{~min}$ (antipode: $10.78 \mathrm{~min}$ ).

Ethyl (1S,2R,3S,5R)-2-benzamido-3,5-diethylcyclopentanecarboxylate ((-)-7): White solid; yield: $90 \%$; $[\alpha]_{\mathrm{D}}^{25}=-2(c=0.235$, EtOH $) ; e e=47 \%$; $t_{\mathrm{R}}=10.65 \mathrm{~min}$ (antipode: $10.04 \mathrm{~min}$ ).

Ethyl (1S,2R,3R,5S)-2-benzamido-3,5-di((E)-styryl)cyclopentanecarboxylate ((+)-6): White solid; yield: $39 \% ;[\alpha]_{\mathrm{D}}^{25}=+38.3(c=0.31, \mathrm{EtOH})$; $e e=96 \% ; t_{\mathrm{R}}=22.22 \mathrm{~min}$ (antipode: $17.28 \mathrm{~min}$ ).

Ethyl (1S,2R,3S,5R)-2-benzamido-3,5-diphenethylcyclopentanecarboxylate $((+)-8)$ : White solid; yield: $71 \% ;[\alpha]_{\mathrm{D}}^{25}=+37(c=0.27, \mathrm{EtOH}) ; e e=96 \%$; $t_{\mathrm{R}}=15.55 \mathrm{~min}$ (antipode: $12.69 \mathrm{~min}$ ).

Ethyl (1S,2R,3R,5S)-2-benzamido-3,5-di(3'-methoxy-3'-oxoprop-1-enyl)cyclopentanecarboxylate $((+)-9)$ : White solid; yield: $75 \% ;[\alpha]_{D}^{25}=+39.3(c=$ $0.385, \mathrm{EtOH}) ; e e=97 \% ; t_{\mathrm{R}}=38.59 \mathrm{~min}$ (antipode: $26.82 \mathrm{~min}$ ).

Ethyl (1S,2R,3R,5S)-2-benzamido-3,5-di((E)-3-oxobut-1-enyl)cyclopentanecarboxylate ((+)-10): White solid; yield: $45 \% ;[\alpha]_{\mathrm{D}}^{25}=+33(c=0.245$, $\mathrm{EtOH}) ; e e=96 \% ; t_{\mathrm{R}}=53.46 \mathrm{~min}$ (antipode: $22.44 \mathrm{~min}$ ).
Ethyl (1S,2R,3S,5R)-2-benzamido-3,5-di(3'-methoxy-3'-oxopropyl)cyclopentanecarboxylate $((+)-11)$ : White solid; yield: $79 \% ;[\alpha]_{\mathrm{D}}^{25}=+44.3(c=$ $0.33, \mathrm{EtOH}) ; e e=97 \% ; t_{\mathrm{R}}=24.46 \mathrm{~min}$ (antipode: $21.34 \mathrm{~min}$ ).

Ethyl (1S,2R,3S,5R)-2-benzamido-3,5-di(3'-oxobutyl)cyclopentanecarboxylate $((+)-12)$ : White solid; yield: $86 \% ;[\alpha]_{\mathrm{D}}^{25}=+35(c=0.375$, EtOH $)$; $e e=96 \% ; t_{\mathrm{R}}=33.02 \mathrm{~min}$ (antipode: $32.62 \mathrm{~min}$ ).

\section{Acknowledgements}

We are grateful to the Hungarian Research Foundation (OTKA nos. NK81371 and K100530) for financial support and acknowledge the receipt of Bolyai János Fellowships for L.K. and E.F.

[1] a) "Synthesis of carbocyclic $\beta$-amino acids": L. Kiss, E. Forró, F. Fülöp in Amino Acids, Peptides and Proteins in Organic Chemistry, Vol. 1 (Ed.: A. B. Hughes), Wiley, Weinheim, 2009, p. 367; b) F. Fülöp, Chem. Rev. 2001, 101, 2181; c) K.-H. Park, M. J. Kurth, Tetrahedron 2002, 58, 8629; d) D. Yang, D.-W. Zhang, Y. Hao, Y.-D. Wu, S.-W. Luo, N.-Y. Zhu, Angew. Chem. 2004, 116, 6887; Angew. Chem. Int. Ed. 2004, 43, 6719; e) N. Rathore, S. H. Gellman, J. J. Pablo, Biophys. J. 2006, 91, 3425; f) J. Mittendorf, F. Kunisch, M. Matzke, H.-C. Militzer, A. Schmidt, W. Schönfeld, Bioorg. Med. Chem. Lett 2003, 13, 433; g) Z. Hamerŝak, M. Roje, A. Avdagic, V. Sunjic, Tetrahedron: Asymmetry 2007, 18, 635; h) S. K. Pandey, G. F. Jogdand, J. C. A. Oliveira, R. A. Mata, P. R. Rajamohanan, C. V. Ramana, Chem. Eur. J. 2011, 17, 12946; i) T. Coursindel, J. Martinez, I Parrot, Eur. J. Org. Chem. 2011, 4519; j) L. Kiss, F. Fülöp, Synlett 2010, 1302; k) F. Csende, F. Fülöp, G. Stájer, Curr. Org. Synth. 2008 5,173 .

[2] a) T. A. Martinek, F. Fülöp, Eur. J. Biochem. 2003, 270, 3657; b) E. Torres, C. Acosta-Silva, F. Rua, A. Alvarez-Larena, T. Parella, V. Branchadell, R. M. Ortuno, Tetrahedron 2009, 65, 5669; c) D. Fernández, E. Torres, F. X. Aviles, R. M. Ortuno, J. Vendrell, Bioorg. Med. Chem. 2009, 17, 3824; d) C. Fernandes, E. Pereira, S. Faure, D. J. Aitken, J. Org. Chem. 2009, 74, 3217; e) F. Fülöp, T. A Martinek, G. K. Tóth, Chem. Soc. Rev. 2006, 35, 323; f) E. A. Porter, B. Weisblum, S. H. Gellman, J. Am. Chem. Soc. 2005, 127, 11516 g) O. Roy, S. Faure, D. J. Aitken, Tetrahedron Lett. 2006, 47, 5981; h) F. Rúa, S. Boussert, T. Parella, I. Diez-Perez, V. Branchadell, E. Giralt, R. M. Ortuno, Org. Lett. 2007, 9, 3643; i) V. D'Elia, H. Zwicknagl, O. Reiser, J. Org. Chem. 2008, 73, 3262; j) C. Fernandes, S. Faure, E. Pereira, V. V. Declerck, R. Guillot, D. J. Aitken, Org. Lett. 2010, 12, 3606; k) S. Chandrasekhar, A. Sudhakar, M. U. Kiran, B. N. Babu, B. Jagadeesh, Tetrahedron Lett. 2008, 49, 7368; 1) A. Hetényi, I. M. Mándity, T. A. Martinek, G. K. Tóth, F. Fülöp, J. Am. Chem. Soc. 2005, 127, 547; m) G. V. M. Sharma, K. S. Reddy, S. J. Basha, K. R. Reddy, A. V. S. Sarma, Org. Biomol. Chem. 2011, 9 , 8102 ; n) S. Celis, E. Gorrea, P. Nolis, O. Illa, R. M. Ortuno, Org Biomol. Chem. 2012, 10, 861; o) E. Szolnoki, A. Hetényi, T. A. Martinek, Z. Szakonyi, F. Fülöp, Org. Biomol. Chem. 2012, 10, 255 p) T. A. Martinek, F. Fülöp, Chem. Soc. Rev. 2012, 41, 687; q) E. Gorrea, P. Nolis, E. Torres, E. Da Silva, D. B. Amabilino, V. Branchadell, R. M. Ortuno, Chem. Eur. J. 2011, 17, 4588.

[3] a) M. Karpf, R. Trussardi, Angew. Chem. 2009, 121, 5871; Angew. Chem. Int. Ed. 2009, 48, 5760; b) N. Satoh, T. Akiba, S. Yokoshima, T. Fukuyama, Tetrahedron 2009, 65, 3239; c) H. Ishikawa, T. Suzuki, Y. Hayashi, Angew. Chem. 2009, 121, 1330; Angew. Chem. Int. Ed. 2009, 48, 1304; d) H. Ishikawa, T. Suzuki, H. Orita, T. Uchimaru, Y. Hayashi, Chem. Eur. J. 2010, 16, 12616; e) J. S. Ko, J. E. Keum, S. Y. Ko, J. Org. Chem. 2010, 75, 7006; f) S. Mohan, S. McAtamney, T. Haselhorst, M. von Itzstein, B. M. Pinto, J. Med. Chem. 2010, 53, 7377; g) A. Kamimura, T. Nakano, J. Org. Chem. 2010, 75, 3133; h) L. D. Nie, X.-X. Shi, K. H. Ko, W.-D. Lu, J. Org. Chem. 2009, 74 3970; i) H. Osato, I. L. Jones, A. Chen, C. L. L. Chai, Org. Lett. 2010, 12, 60; j) B. Sullivan, I. Carrera, M. Drouin, T. Hudlicky, 
Angew. Chem. 2009, 121, 4293; Angew. Chem. Int. Ed. 2009, 48, 4229; k) B. M. Trost, T. Zhang, Angew. Chem. 2008, 120, 3819; Angew. Chem. Int. Ed. 2008, 47, 3759; 1) S. Zhu, S. Yu, Y. Wang, D. Ma, Angew. Chem. 2010, 122, 4760; Angew. Chem. Int. Ed. 2010, 49, 4656; m) J. Calveras, Y. Nagai, I. Sultana, Y. Ueda, T. Higashi, M. Shoji, T. Sugai, Tetrahedron 2010, 66, 4284.

[4] a) T. Honda, S. Kubo, T. Masuda, M. Arai, Y. Kobayashi, M. Yamashita, Bioorg. Med. Chem. Lett. 2009, 19, 2938; b) J.-F. Soulé, A. Mathieu, S. Norsikian, J.-M. Beau, Org. Lett. 2010, 12, 5322; c) W.-H. Wen, S.-Y. Wang, K.-C. Tsai, Y.-S. E. Cheng, A.-S. Yang, J.-M. Fang, C.-H. Wong, Bioorg. Med. Chem. 2010, 18, 4074; d) G. Xu, M. J. Kiefel, J. C. Wilson, P. W. Andrew, M. R. Oggioni, G. L. Taylor, J. Am. Chem. Soc. 2011, 133, 1718; e) W. J. Lü, Y. L. Chen, W. P. Ma, X. Y. Zhang, F. Luan, M. C. Liu, X. G. Chen, Z. D. Hu, Eur. J. Med. Chem. 2008, 43, 569; f) J. Li, M. Zheng, W. Tang, P.-L. He, W. Zhu, T. Li, J.-P. Zuo, H. Liu, H. Jiang, Bioorg. Med. Chem. Lett. 2006, 16, 5009 ; g) N. T. Kipassa, H. Okamura, K. Kina, T. Hamada, T. Iwagawa, Org. Lett. 2008, 10, 815; h) M. Weïwer, C.-C. Chen, M. M. Kemp, R. J. Linhardt, Eur. J. Org. Chem. 2009, 2611; i) P. G. Wyatt, B. A. Coomber, D. N. Evans, T. I. Jack, H. E. Fulton, A. J. Wonacott, P. Colman, J. Varghese, Bioorg. Med. Chem. Lett. 2001, 11, 669; j) A. Albohy, S. Mohan, R. B. Zheng, B. M. Pinto, C. W. Cairo, Bioorg. Med. Chem. 2011, 19, 2817; k) H. Ishikawa, B. P. Bondzic, Y. Hayashi, Eur. J. Org. Chem. 2011, 6020; 1) J.-J. Shie, J.-M. Fang, P.-T. Lai, W.-H. Wen, S.-Y. Wang, Y.-S. E. Cheng, K.-C. Tsai, A.-S. Yang, C.-H. Wong, J. Am. Chem. Soc. 2011, 133, 17959; m) J. Magano, Tetrahedron 2011, 67,7875 .

[5] a) P. Chand, Y. S. Babu, S. Bantia, S.; Rowland, A. Dehghani, P. L. Kotian, T. L. Hutchison, S. Ali, W. Brouillette, Y. El-Kattan, T.-H. Lin, J. Med. Chem. 2004, 47, 1919; Rowland, A. Dehghani, P. L. Kotian, T. L. Hutchison, S. Ali, W. Brouillette, Y. El-Kattan, T.-H. Lin, J. Med. Chem. 2004, 47, 1919; b) A. J. Oakley, S. Barrett, T. S. Peat, J. Newman, V. A. Streltsov, L. Waddington, T. Saito, M. Tashiro, J. L. McKimm-Breschkin, J. Med. Chem. 2010, 53, 6421; c) P. Chand, S. Bantia, P. L. Kotian, Y. El-Kattan, T.-H. S. Lin, Y. S. Babu, Bioorg. Med. Chem. Lett. 2005, 15, 4071; d) Y. Cui, Z. Jiao, J. Gong, Q. Yu, X. Zheng, J. Quan, M. Luo, Z. Yang, Org. Lett. 2010, 12, 4; e) X. Yi, Z. Guo, F. M. Chu, Bioorg. Med. Chem. 2003, 11, 1465 ; f) C. M. Bromba, J. W. Mason, M. G. Brant, T. Chan, M. D. Lunke, M. Petric, M. J. Boulanger, J. E. Wulff, Bioorg. Med. Chem. Lett. 2011, 21, 7137.

[6] a) I. Atodiresei, I. Schiffers, C. Bolm, Chem. Rev. 2007, 107, 5683; b) F. Fülöp, M. Palkó, E. Forró, M. Dervarics, T. A. Martinek, R. Sillanpää, Eur. J. Org. Chem. 2005, 3214; c) Z. Szakonyi, S. Gyónfalvi, E. Forró, A. Hetényi, N. De Kimpe, F. Fülöp, Eur. J. Org. Chem. 2005, 4017; d) M. Palkó, E. Sándor, P. Sohár, F. Fülöp, Monatsh. Chem. 2005, 136, 2051; e) G. Benedek, M. Palkó, E. Wéber, T. A. Martinek, E. Forró, F. Fülöp, Eur. J. Org. Chem. 2008, 3724; f) S. Kobayashi, K. Kamiyama, M. Ohno, J. Org. Chem. 1990, 55, 1169; g) O. Songis, C. Didierjean, J. Martinez, M. Calmes, Tetrahedron: Asymmetry 2008, 19, 2135; h) L. Kiss, I. Szatmári, F. Fülöp, Lett. Org. Chem. 2006, 3, 463; i) Y. Matsushima, J. Kino, Eur. J. Org. Chem. 2009, 1619; j) L. Kiss, E. Forró, S. Fustero, F. Fülöp, Org. Biomol. Chem. 2011, 9, 6528; k) L. Kiss, E. Forró, S. Fustero, F. Fülöp, Eur. J. Org. Chem. 2011, 4993; l) E. Forró, L. Schönstein, L. Kiss, A. Vega-Peñaloza, E. Juaristi, F. Fülöp, Molecules 2010, 15, 3998.

[7] C. L. Campbell, C. Hassler, S. S. Ko, M. E. Voss, M. A. Guaciaro, P. H. Carter, R. J. Cherney, J. Org. Chem. 2009, 74, 6368.
[8] a) M. E. Bunnage, T. Ganesh, I. B. Masesane, D. Orton, P. G. Steel Org. Lett. 2003, 5, 239; b) I. B. Masesane, P. G. Steel, Tetrahedron Lett. 2004, 45, 5007; c) J. Chola, I. B. Masesane, Tetrahedron Lett. 2008, 49, 5680; d) L. Kiss, E. Forró, F. Fülöp, Tetrahedron Lett. 2006, 47, 2855; e) L. Kiss, E. Forró, T. A. Martinek, G. Bernáth, N. De Kimpe, F. Fülöp, Tetrahedron 2008, 64, 5036; f) L. Kiss, E. Forró, R. Sillanpää, F. Fülöp, J. Org. Chem. 2007, 72, 8786; g) L. Kiss, E. Forró, R. Sillanpää, F. Fülöp, Synthesis 2010, 153; h) L. Kiss, E. Forró, R. Sillanpää, F. Fülöp, Tetrahedron: Asymmetry 2008, 19, 2856 ; i) L. Kiss, E. Forró, F. Fülöp, Tetrahedron 2012, 68, 4438; j) L. Kiss, E. Forró, F. Fülöp, Lett. Org. Chem. 2011, 8, 220; k) L. Kiss, E. Forró, R. Sillanpää, F. Fülöp, Tetrahedron 2010, 66, 3599.

[9] a) P. Wipf, X. Wang, Tetrahedron Lett. 2000, 41, 8747; b) G. Benedek, M. Palkó, E. Wéber, T. A. Martinek, E. Forró, F. Fülöp, Tetrahedron: Asymmetry 2009, 20, 2220; c) L. Kiss, B. Kazi, E. Forró, F. Fülöp, Tetrahedron Lett. 2008, 49, 339; d) B. Kazi, L. Kiss, E. Forró, F. Fülöp, Tetrahedron Lett. 2010, 51, 82; e) B. Kazi, L. Kiss, E. Forró, I. Mándity, F. Fülöp, Arkivoc 2010, (ix), 31.

[10] a) L. Kiss, M. Nonn, E. Forró, R. Sillanpää, F. Fülöp, Tetrahedron Lett. 2009, 50, 2605; b) M. Nonn, L. Kiss, E. Forró, Z. Mucsi, F. Fülöp, Tetrahedron 2011, 67, 4079; c) M. Nonn, L. Kiss, R. Sillanpää, F. Fülöp, Beilstein J. Org. Chem. 2012, 8, 100.

[11] A. Mondière, R. Peng, R. Remuson, D. J. Aitken, Tetrahedron 2008 , 64, 1088.

[12] T. J. Peelen, Y. Chi, E. P. English, S. H. Gellman, Org. Lett. 2004, 6 , 4411.

[13] A. E. Nadany, J. E. Mckendrick, Synlett 2006, 2139.

[14] a) S. Fustero, J. F. Sanz-Cervera, J. L. Acena, M. Sanchez-Rosello, Synlett 2009, 525; b) S. Fustero, M. Sanchez-Rosello, V. Rodrigo, C. Del Pozo, J. F. Sanz-Cervera, A. Simon, Org. Lett. 2006, 8, 4129; c) S. Fustero, M. Sanchez-Rosello, J. F. Sanz-Cervera, J. L. Acena, C. Del Pozo, B. Fernandez, A. Bartolome, A. Asensio, Org. Lett. 2006, 8,4633 ; d) S. Fustero, M. Sanchez-Rosello, J. L. Acena, B. Fernandez, A. Asensio, J. F. Sanz-Cervera, C. Del Pozo, J. Org. Chem. 2009, 74, 3414

[15] a) M. E. Bunnage, A. M. Chippindale, S. G. Davies, R. M. Parkin, A. S. Smith, J. M. Withey, Org. Biomol. Chem. 2003, 1, 3698; b) S. A. Davies, A. C. Garner, M. J. C. Long, R. M. Morrison, P. M Roberts, E. D. Savory, A. D. Smith, M. J. Sweet, J. M. Withey, Org. Biomol. Chem. 2005, 3, 2762; c) E. Abraham, S. G. Davies, A. J. Docherty, K. B. Ling, P. M. Roberts, A. J. Russel, J. E. Thomson, S. M. Toms, Tetrahedron: Asymmetry 2008, 19, 1356; d) S. G. Davies, M. J. Durbin, S. J. S. Hartman, A. Matsuno, P. M. Roberts, A. J. Russell, A. D. Smith, J. E. Thomson, S. M. Toms, Tetrahedron: Asymmetry 2008, 19, 2870; e) E. Abraham, T. D. W. Claridge, S. G. Davies, B. Odell, P. M. Roberts, A. J. Russell, A. D. Smith, L. J. Smith, H. R. Storr, M. J. Sweet, A. L. Thompson, J. E. Thomson, G. E. Tranter, D. J. Watkin, Tetrahedron: Asymmetry 2011, 22, 69; f) F. CharnayPouget, M. Frank, J.-P. Baltaze, E. Pereira, D. J. Aitken, Arkivoc 2012, (v), 80.

[16] a) Y. Fricke, N. Kopp, B. Wünsch, Synthesis 2010, 791; b) M. Lee, S. S. Stahl, S. H. Gellman, Org. Lett. 2008, 10, 5317; c) G. Malik, X Guinchard, D. Crich, Org. Lett. 2012, 14, 596.

[17] E. Forró, F. Fülöp, Tetrahedron: Asymmetry 2008, 19, 1005.

[18] E. Forró, F. Fülöp, Tetrahedron: Asymmetry 2004, 15, 573.

[19] E. Forró, J. Chromatogr. A 2009, 1216, 1025

Received: September 7, 2012

Revised: November 13, 2012 Published online: December 19, 2012 